\title{
perifèria
}

Número 3, Diciembre 2005

www.periferia.name

\section{Redes sociales, apoyo social y salud}

\author{
Roser Fernández Peña - Antropología Social y Cultural, UAB ${ }^{1}$
}

\section{Resumen}

En los últimos años, el ambiente social ha sido reconocido como un complejo sistema estructural, cultural, interpersonal y psicológico con propiedades adaptativas y de ajuste frente a determinadas situaciones relacionadas con los procesos de salud-enfermedad y también como un sistema contenedor de elementos estresares y/o amortiguadores del mismo. Ello justifica un creciente interés hacia los recursos derivados de los vínculos sociales de los individuos así como la relación existente entre las relaciones interpersonales y la salud de las personas.

De este modo, conceptos como "redes sociales,", "sistemas de apoyo comunitario", "apoyo social" (social support), o "recursos del entorno", han sido conceptos clave para el desarrollo de las líneas teóricas que han guiado las investigaciones que relacionan las redes sociales y la salud.

Este artículo bibliográfico elaborado a partir de un trabajo de curso en el marco de los estudios de doctorado pretende ser una aproximación al desarrollo conceptual y empírico existente tanto desde el punto de vista antropológico como sanitario.

\begin{abstract}
In recent years the social environment has been recognized as a structural, cultural, interpersonal and psychologically complex system with adaptive and adjustment properties in situations related to the processes of health and disease and also as a system which contains stressful and buffer elements.

It justifies an increasing interest towards the resources derived from the individual social bonds as well as the interpersonal relations and the health of the people.

Thus, concepts like "social networks", "systems of community support", "social support" or "resources of the environment", have been key concepts for the development of the theoretical lines that have guided the investigations that relate the social networks and health.

This article, elaborated in the framework of the doctorate studies, attempts to be an approach to the conceptual and empirical development, from the anthropological and public health viewpoint.
\end{abstract}

\footnotetext{
${ }^{1}$ Enviar correspondencia a: Roser Fernández [roserferpe@hotmail.com].
} 


\section{perifèria}

Número 3, Diciembre 2005

www. periferia. name

\section{I ntroducción}

Desde una perspectiva holística y lejos de un enfoque biologista de los procesos de salud y enfermedad, el tema principal de este artículo, se suma a la consideración de la dimensión social de la salud definida por la OMS:

La salud es un estado de completo bienestar físico, mental y social, y no consiste solamente en la ausencia de enfermedades. La posesión del mejor estado de salud que se es capaz de conseguir constituye uno de los derechos fundamentales de todo ser humano, cualquiera que sea su raza, religión, ideología política y condición económico-social (Carta Fundacional de la OMS, 1948).

Es así como aspectos relativos al grado de integración o aislamiento en relación al medio social que rodea al individuo, ha motivado el desarrollo de teorías e investigaciones asociándolos a determinados problemas de salud.

\section{Los inicios teóricos del medio ambiente social y la salud}

Las primeras teorías sobre integración social tienen su origen en el campo de la sociología con Émile Durkheim y psicoanalistas como J ohn Bowly.

El trabajo de Durkheim resultó pionero en los trabajos que relacionan la cohesión e integración social con la salud. En El suicidio (1897) el autor demuestra cómo los hechos sociales pueden ser utilizados para explicar los patrones cambiantes de la tendencia hacia el suicidio. En su obra realiza un meticuloso estudio cuantitativo de los índices de suicidios en diferentes segmentos de la población de Europa con el fin de refutar las teorías que pretenden explicar las variaciones de grupo en el número de suicidios en base de factores psicológicos, biológicos, raciales, genéticos, climáticos o geográficos y también para apoyar con pruebas empíricas su propia explicación sociológica:

(...) resulta que la cifra social de los suicidios no se explica más que sociológicamente. Es la constitución moral de la sociedad la que fija en cada instante el contingente de las muertes voluntarias. Existe pues, para cada pueblo, una fuerza colectiva, de una energía determinada, que 


\section{perifèria}

Número 3, Diciembre 2005

www. periferia. name

impulsa a los hombres a matarse. Los actos que el paciente lleva a cabo y que, a primera vista parecen expresar tan solo su temperamento personal, son en realidad la consecuencia y prolongación de un estado social que ellos manifiestan exteriormente (Durkheim, 1982: 326).

Johan Bowly, uno de los más importantes psiquiatras del siglo XX, formuló la attachment theory, en la que relaciona el medio ambiente y la patología, en concreto mediante el estudio de los mecanismos mediante los cuales el medio contribuye al desarrollo de la génesis de la neurosis en la primera infancia. Bowly defiende que el primer motivo por el que los niños necesitan la psicoterapia es la separación de éstos de sus madres, hecho que cataloga como insaludable. Todo ello está en consonancia con la idea de que existe una necesidad humana universal de vínculos afectivos cuyos lazos íntimos creados en la infancia formarían una sólida base emocional para la vida adulta, además de proporcionar unos prototipos para las relaciones sociales posteriores. De este modo, la experiencia emocional de la primera infancia aparece como un período crítico de desarrollo no sólo desde el punto de vista emocional y cognitivo sino también para la salud en general.

Secure attachment, provides an external ring of psychological protection which maintains the child's metabolism in a stable state, similar to internal homeostasis mechanism of blood pressure and temperature control (Bowly, J. 1969, citado en Berckman, L.F. y col. 2000:844-845).

Pero es a partir de la década de los setenta cuándo se advierte un interés creciente hacia las dimensiones derivadas de las relaciones sociales, hecho que se refleja con un más que significativo aumento de trabajos encabezados bajo el término social support publicados en el Social Citation Index (que pasó de 2 artículos en 1972 a 50 en 1982).

Buena parte de este interés es debido a las innovadoras formulaciones teóricas de Cassel y Caplan, al permitir conceptualizar los recursos derivados de las relaciones sociales y establecer estrategias de intervención comunitaria dirigidas a mejorar los sistemas de apoyo. 


\section{perifèria}

Número 3, Diciembre 2005

www. periferia.name

En síntesis, las ideas formuladas por J. Cassel (1974) sostienen que los cambios en el ambiente social pueden alterar la resistencia de los individuos hacia la enfermedad, debido a las alteraciones metabólicas que desencadenan ya que la falta de señales o de información relevante por parte de relaciones sociales (en especial las referidas a personas significativas y próximas para el individuo), puede constituir el origen de la enfermedad.

En otro sentido, la aportación de G. Caplan (1974) se dirige a subrayar la importancia que tiene para el bienestar de los individuos los recursos derivados de las distintas relaciones sociales mantenidas tales como apoyo emocional, instrumental... Entre sus contribuciones, destaca la necesidad de realización de actividades que deberían realizar profesionales para favorecer el desarrollo de contactos sociales proveedores de apoyo en una determinada comunidad.

Contando las aportaciones de Cassel y Caplan, para Gottlieb (1981), uno de los motivos que justifican el aumento de los estudios de la salud y el ambiente social es el origen de una perspectiva ecológico-comunitaria dentro de la Psicología cuya prioridad se centraría en aislar y manipular factores ambientales con el fin de favorecer una mayor prevalencia de la salud dentro de la población.

Es de destacar en esta línea el trabajo pionero de Faris y Dunham (1939) quienes trabajan sobre los correlatos ecológicos de los trastornos mentales en Chicago al estudiar la distribución temporo-espacial que seguía la incidencia y prevalencia de la psicosis en la ciudad. Sus contribuciónes más relevantes consistieron en demostrar la asociación entre trastornos psiquiátricos y zonas de desorganización social, donde existiría mayor confusión sobre las normas de conducta y sensación de futilidad sobre la propia habilidad para obtener apoyo y reconocimiento. Pocos años antes, Faris (1934) ya había formulado la social isolation hipothesis al sostener que la interacción social era esencial para el desarrollo de una personalidad normal y promulgar una conducta social apropiada. En este sentido, sus trabajos sugieren que el aislamiento social de los individuos constituye el mayor riesgo para desarrollar un desorden mental. 


\section{perifèria}

Número 3, Diciembre 2005

www. periferia. name

Otro de los motivos señalados por Gottlieb que justifican el aumento de estos estudios son los hallazgos encontrados en trabajos realizados entre la población americana en los que se ponía de manifiesto la importancia de las "redes informales de apoyo" (amigos, vecinos, etc.) para la salud mental de la población, al demostrar cómo las personas afectadas por problemas emocionales, situaciones difíciles y/o generadoras de estrés, elegían a personas de su entorno antes que a especialistas en salud mental, como psiquiatras o psicólogos, para intentar solucionarlos. Este hecho otorga el reconocimiento a las personas que pertenecen al entorno próximo del individuo (con formación profesional o no) como "fuentes proveedoras de apoyo" para el mantenimiento de la salud mental dentro de la comunidad.

\section{Los roles sociales y la salud}

Otra de las variables estudiadas en el marco de las relaciones sociales y que ha sido motivo de controversia a la luz de investigaciones de diferentes autores, ha sido el desempeño o no de múltiples roles sociales.

Así, algunos sociólogos sugieren que la participación en múltiples dominios sociales son perjudiciales para el bienestar psicológico al contemplar el medio ambiente social de las personas como juegos de relaciones de rol interrelacionadas en los que el desempeño de cada uno de los roles estaría inevitablemente asociado a un conjunto diferente de obligaciones conflictivas con la consecuente experiencia negativa de estrés. Así, el término role strain, fue acuñado por Goode (1960) para describir la complicada dificultad para llevar a cabo de manera adecuada el desempeño de múltiples roles simultáneamente.

Años más tarde y con un limitado soporte desde el punto de vista empírico, las ideas de Goode fueron sustituidas por Sieber (1974) quien sustenta la idea que la posesión de múltiples roles es beneficiosa para el bienestar psicológico. Su argumento apunta más bien a que la recompensa que reporta el hecho de tener múltiples roles, excede los inconvenientes asociados al role strain. Además, incide 


\section{perifèria}

Número 3, Diciembre 2005

www. periferia. name

en la necesidad de prestar atención a aquellos mecanismos que favorecerían la acumulación de roles considerándola como psicológicamente deseable y socialmente normales.

Siguiendo la misma línea, Marks (1977) elabora la role accumulation theory, haciendo hincapié en que la acumulación de rol es mas gratificante que estresante al equipararla con una potencial fuente de salud, prestigio y autoimagen favorable.

Thoits (1983), elabora la identity accumulation hipothesis argumentando que las identidades de las personas están relacionadas con los roles que desempeñan en la sociedad. Sugiere que las identidades de rol proporcionan a las personas información acerca de lo que ellos son en su existencia. Los roles sociales, en algún sentido, proporcionan un motivo para vivir. “... if one knows who one is (in a social sense), then one knows how to behave" (Thoits, 1983: 175).

Así, a medida que las personas acumulan identidades de rol, poseen un sentido significativo de sus vidas. Ello implica que el sentido y significado que uno tiene de la vida es un componente integral para el bienestar psicológico y que una falta de sentido de la propia existencia a menudo conduce a conductas inapropiadas y a un comportamiento autodestructivo.

\section{Comportamientos en salud y redes sociales}

La Theory of Planned Behaviour (TPB) es una extensión de la Theory of Reasoned Action (TRA), y ambas se han desarrollado y utilizado ampliamente en las investigaciones del comportamiento en el terreno de la salud, específicamente en torno al HIV/SIDA.

Según esta teoría, el comportamiento relativo a la salud (Behavioural intention), está determinado por:

- Las actitudes hacia el comportamiento, determinadas por la creencia que un comportamiento específico puede tener unas consecuencias concretas así 


\section{perifèria}

Número 3, Diciembre 2005

www. periferia. name

como la valoración o evaluación de dichas consecuencias.

- Las normas subjetivas o la creencia que otras personas relevantes para ego aprobarán su comportamiento, a lo que hay que sumar la motivación personal que puede suponer el hecho de satisfacer las expectativas de otros.

- El control percibido del comportamiento, determinado por la creencia sobre el acceso a los recursos para actuar con éxito, a lo que hay que añadir el éxito percibido proveniente de dichos recursos (información, habilidades, dependencia o independencia de otros...).

- Variables socio-demográficas y rasgos de personalidad que condicionan actitudes, normas subjetivas y control del comportamiento percibido.

El trabajo de Umberson (1987), refleja una compleja interrelación entre variables como el género, el status marital (con sus roles correspondientes), la familia y los hábitos de salud que - mediada por complejos procesos sociales -, influiría en la salud de la persona.

Así, la influencia del control social en los hábitos de salud se ejercería en dos sentidos. Indirectamente, a través de la adopción de hábitos de salud bajo las ideas de que por un lado, el individuo es más propenso a adoptar determinados estilos de vida saludables si tiene con quién compartir esos hábitos y por otro lado la internalización de normas de conducta, por ejemplo a través del marco de referencia y modelo a seguir que ofrece la familia por lo que respecta a los hábitos de salud. En otro sentido y directamente, el control social se ejercería mediante regulaciones, sanciones o intervención física como sería la participación sin mediaciones en el control de la alimentación, la preparación y/o administración de tratamientos.

\section{Redes sociales, soporte social y salud}

Uno de los recursos derivados de las relaciones sociales y de relevante importancia en contextos relacionados con los procesos de salud - enfermedad, es el apoyo 


\section{perifèria}

Número 3, Diciembre 2005

www. periferia. name

social (social support).

Una de las características de esta línea de investigación es la diversa conceptualización existente sobre el término en función de sus diferentes dominios, hecho que ha dado lugar a un importante contingente de estudios realizados con enfoques y técnicas muy diversas y por tanto, con una dificultosa evaluación y comparación de resultados. En general, parece aceptado el estudio del soporte social desde tres perspectivas: estructural, funcional y contextual.

Desde un punto de vista estructural, Lin et al. (1979, 1981), definen el apoyo social como provisiones instrumentales o expresivas, reales o percibidas, aportadas por la comunidad, redes sociales y amigos íntimos. En su definición, reflejan los tres niveles de análisis necesarios para su estudio, si bien a la hora de obtener apoyo, los tres niveles están interconectados.

El primer nivel de análisis estaría ocupado por la comunidad, haciendo referencia a la integración social, regulación normativa y orden social que ésta ofrece dando significado a la propia existencia y protegiendo a la persona contra la incertidumbre, que en último extremo daría lugar a un funcionamiento desordenado.

En un segundo nivel de análisis lo constituyen las redes sociales, que aportaría un sentido de relaciones y de unión con los demás.

El tercer nivel de análisis sería el correspondiente al más íntimo, al que proporciona un sentimiento de vínculo más estrecho, en el cual se esperan intercambios recíprocos y mutuos y también en el que se comparte una responsabilidad por el bienestar de los otros.

Desde la perspectiva estructural, se estudia el campo social en el que está inmerso el sujeto siendo la red social la unidad de análisis del individuo. Desde este enfoque se asume que: los beneficios de las redes son directamente proporcionales al tamaño de las mismas y que tener una relación es equivalente a obtener apoyo de la misma. Sin embargo, es necesario señalar que tales afirmaciones son 


\section{perifèria}

Número 3, Diciembre 2005

www. periferia.name

cuestionables ya que obvian la posibilidad de la existencia de conflictos asociados a las relaciones sociales y a la polémica pero consistente distinción entre las dimensiones objetivo/subjetivo del apoyo.

De este modo, autores como Cassel y Cobb conceptualizan el apoyo social en términos cognitivos, tomando en consideración la dimensión subjetiva del mismo (apoyo percibido), ya que es esta percepción precisamente, la que se considera promotora de la salud. La importancia de esta distinción, radica en que algunas personas reciben apoyo y no lo perciben, y en ausencia de percepción de un apoyo disponible, éste no podrá ser utilizado.

Siguiendo con la perspectiva estructural de las redes sociales, las dimensiones estudiadas más importantes y que guardan relación con la salud son:

- Tamaño: Referido al número de personas que componen la red social, o número de sujetos con los que mantiene contacto personal el sujeto central. En general, esta variable se relaciona positivamente con el bienestar y la salud. Algunos autores, han encontrado diferencias significativas entre el tamaño de la red social en sujetos que padecen algún tipo de trastorno mental siendo en general menores en comparación con otros sujetos sin estos problemas de salud.

- Densidad: Interconexión entre los miembros independientemente del sujeto central. En este punto, los resultados son contradictorios ya que no puede establecerse una densidad ideal al depender ésta de otras variables como son los propios sujetos, sus necesidades y su situación concreta. Algunas investigaciones otorgan a las redes sociales más densas una mayor capacidad para proveer apoyo emocional, si bien su aceptabilidad dependerá de los sujetos y situaciones. Por otro lado, las redes sociales densas, no serían adecuadas en determinadas situaciones como la viudedad ya que impiden la creación de nuevos contactos sociales y perpetúan de este modo, el modelo social previo. 


\section{perifèria}

Número 3, Diciembre 2005

www. periferia. name

- Reciprocidad: Grado en que los recursos de la red son intercambiados equitativamente entre las partes, al equilibrio o desequilibrio del intercambio en la relación entre dos personas. Los resultados demuestran que las relaciones recíprocas promueven más la salud que aquéllas en las que el sujeto central inicia siempre el contacto. Algunas investigaciones, registran un gran número de relaciones desequilibradas y no recíprocas en pacientes con problemas de salud mental.

- Parentesco: Variable referida a si la red está formada en su mayoría por parientes o no y que resulta ser predictora de un buen ajuste ante las crisis vitales. En algunos trabajos, el número de no parientes en la red se ha relacionado positivamente con estados de felicidad bajo la idea que estas relaciones, serían elegidas libremente.

- Homogeneidad: Semejanza o congruencia entre los miembros de una red en una dimensión determinada: actitudes, experiencia, valores, etc.. Algunos autores señalan que las personas que forman parte de redes homogéneas, tienen interacciones más reforzantes.

La importancia de la perspectiva estructural en el estudio del apoyo social, radica en la información que ofrece acerca de la disponibilidad potencial del apoyo. Sin embargo, la existencia de una relación social no puede equipararse a obtener apoyo de la misma. Algunos autores señalan que para que una red social sea efectiva, no sólo es necesario que exista desde un punto de vista estructural sino también que las personas/nodos que formen parte de la red, tengan la habilidad, conocimiento y motivación suficiente para proveer soporte, de lo cual se sigue que no todos los nodos serían "operativos" para la provisión del mismo. Y es que en este sentido, parece ser que aquellos acontecimientos vitales inesperados como por ejemplo la aparición de la enfermedad en algunos casos, resultan ser más problemáticos desde el punto de vista de la efectividad del soporte, ya que además de que el sujeto está sometido a mayor nivel de estrés porque no ha podido prepararse para el evento, a menudo los miembros de la red social no ofrecen el apoyo adecuado por falta de 


\section{perifèria}

Número 3, Diciembre 2005

www. periferia. name

habilidades y/o conocimiento a pesar de que exista motivación para ello.

En el abordaje del soporte social desde un punto de vista funcional, destaca la aportación de House (1981), uno de los autores que ha desarrollado más extensamente el apoyo social como concepto, considerándolo como una transacción interpersonal que engloba uno o más de los siguientes aspectos: emocional, instrumental, información y valoración (información relevante para la autoevaluación). No obstante, y a pesar de las diferentes clasificaciones, la mayoría de autores coinciden en destacar tres funciones básicas: apoyo emocional, material e instrumental.

El enfoque funcional del apoyo social, se centra por tanto en los aspectos cualitativos del mismo poniendo el acento en las funciones y los recursos que se pueden obtener como resultado de las relaciones sociales. De entre los recursos intercambiados, destaca el feedback, la asistencia conductual, el dinero y pequeños servicios.

De entre las funciones relacionadas con la salud, una clasificación realizada por Shumaker y Brownell (1984) distingue entre:

1. Funciones del apoyo social sustentadoras de la salud. Referidas principalmente a situaciones con ausencia de estrés y cuya función principal va dirigida a la promoción de la salud a través del mantenimiento y fomento de la autoidentidad, la autoestima, y la gratificación de necesidades afiliativas.

2. Funciones del apoyo social reductoras del estrés. Aludiendo a la función que desempeña ante situaciones estresantes como es el caso de la presencia de enfermedad, a través de dos funciones específicas: la evaluación y la adaptación cognitiva. En la primera, el apoyo social puede cambiar la interpretación primaria que se haga del agente estresor así como cambiar la evaluación de los recursos de afrontamiento con los que se cuenta para hacerle frente. En cuanto a la segunda, el apoyo social puede proporcionar 


\section{perifèria}

Número 3, Diciembre 2005

www. periferia. name

información sobre métodos para controlar la amenaza.

Algunas consideraciones metodológicas en el estudio del apoyo social, merecen especial importancia una vez vistas las perspectivas estructurales y funcionales del mismo. Una de ellas sería evitar la confusión entre la estructura de la red del individuo con la funcionalidad de la misma ya que puede ser que existan diferencias entre estructura y función. En otro sentido, la interpretación de algunos resultados debe considerar la posibilidad de que exista cierta reversibilidad causal entre apoyo social y problemas de salud en los que los últimos tendrían un impacto sobre el apoyo social real o percibido más que a la inversa. Éste es el caso de personas gravemente enfermas que plantean exigencias excesivas o los casos de patología mental. Por último, otras investigaciones sobre apoyo social, tienen en cuenta otras variables como son el tipo de personalidad de los sujetos de estudio y el locus de control (interno o externo) sobre diferentes situaciones y procesos, hecho que obliga a considerar otros factores que podrían intervenir en su evaluación.

Por último, y desde una perspectiva contextual, es necesario considerar los aspectos situacionales en los que el apoyo es percibido, movilizado, aportado o recibido, todo ello con el fin de relacionar con un carácter predictivo, el apoyo social con el bienestar de las personas.

Por ello, es necesario considerar las características de los participantes en el apoyo tanto del receptor como del donante por su influencia en el mismo, así como la duración del apoyo.

En cuanto a las primeras, parece existir variación entre la fuente de procedencia del apoyo en función de los sujetos de manera que el apoyo de los compañeros de trabajo tiene mejores consecuencias en varones que en mujeres, mientras que el apoyo familiar es más importante ante problemas relacionados con la salud frente al procedente de los amigos. En otro sentido, características del receptor como la personalidad, roles, presencia o no de otros recursos y/o la creencia acerca del momento en el que es adecuado aceptar la ayuda juegan un papel relevante para la efectividad del soporte. Por último, en situación de enfermedad el apoyo está unido 


\section{perifèria}

Número 3, Diciembre 2005

www. periferia. name

y en relación con las diferentes fases del afrontamiento ${ }^{2}$ de manera que un apoyo puede ser adecuado o no en función del momento en el que se preste y perciba como tal.

En cuanto a la duración del apoyo social en el tiempo, éste cobra especial importancia ante cierto tipo de problemas como es el caso de pacientes crónicos o el de personas que sufren estrés a largo plazo, ya que exige una habilidad adecuada para mantener y/o cambiar el apoyo a lo largo del tiempo por parte de los donantes del apoyo

\section{La intervención en salud desde las redes sociales}

Desde el punto de vista de la Salud, cuando los profesionales utilizan o hacen referencia a la red social, se centran en los vínculos sociales con los que cuenta un individuo.

A través del análisis de la red social, se persigue abarcar una amplia gama de las relaciones sociales prestando atención a múltiples aspectos y efectos de esas relaciones, además de proporcionar un método para describir los caracteres estructurales de los vínculos sociales y analizar los distintos patrones de interacción. Desde el enfoque funcional de las redes sociales, el apoyo social se ha considerado como una función relacionada con el funcionamiento psicológico de los individuos, que es vehiculizada en el interior del marco estructural de la red.

En nuestro contexto actual y desde la Salud Pública conceptos como la prevención, promoción y rehabilitación de la salud, sitúan en un lugar privilegiado a las personas, familias y a la propia comunidad como recursos naturales/informales disponibles que garantizan la continuidad de los servicios profesionales en salud. Paralelamente, en los últimos años se han implantado grupos organizados de

\footnotetext{
${ }^{2}$ El afrontamiento se define como el esfuerzo individual que el paciente con problemas de salud realiza y cuyo propósito es la reducción de los estresares fisiológicos y psicológicos a grados tolerables para adaptarse a la situación de estrés.
} 


\section{perifèria}

Número 3, Diciembre 2005

www. periferia. name

personas como los grupos de autoayuda ${ }^{3}$ cuyo papel principal es el de facilitar estrategias adaptativas frente a la enfermedad.

Además en determinados periodos del ciclo vital como la ancianidad, la relación entre los individuos y los grupos sociales a los que pertenecen, permiten movilizar recursos psicológicos y materiales frente a situaciones indeseables como la incapacidad, soledad y/o enfermedad.

Desde el punto de vista de la intervención comunitaria en Salud, es necesario conocer la estructura social y los diferentes niveles de intervención comunitaria con el objetivo de potenciar, crear o complementar recursos que proporcionen apoyo social tanto a nivel individual-familiar como colectivo.

\section{Bibliografía}

Barrón López de Roda, A. (1989) “ Estrés psicosocial, apoyo social y depresión en mujeres. Un estudio empírico en Aranjuez", Universidad Complutense de Madrid. Colección tesis doctorales no 44/89

Barrón López de Roda, A (1990). "Perspectivas de estudio en el apoyo social", JANO 9-15. Vol. XXXVIII № 898 pp. 62-73.

Berckman, L.F; Glass, T.; Brissette, I.; Seeman,T.E. (2000). "From social integration to health: Durkheim in the new millennium". Social Science \& Medicine 51 pp. 843-57.

Bowly, J. (1969). Attachment and loss. London: Hogarth Press

Cassel,J (1974). "Psychosocial Processes and Stress. Theoretical Formulations". International of Health Services, 6 pp.471-482.

\footnotetext{
${ }^{3}$ El modelo de autoatención en salud, concretamente en el problema del alcoholismo, ha sido estudiado ampliamente por Menéndez, E.L. (1984).
} 


\section{perifèria}

Número 3, Diciembre 2005

www. periferia.name

Caplan, H (1974) "Systems and Community Mental Health. Behavioral Publications. New York.

Cohen, S.; Brissette,I. "Social integration and health: the case of the common cold" Joss, Volume 1. http://www.heinz.cmu.edu/project/INSA/joss/sih.html (Consulta febrero 2005).

Cohen, S.; Syme, S.L. (1985). Social support and health. Academic Press, INC

Diaz Veiga, Purificación (1987). “Evaluación del apoyo social” en Fernández Ballesteros “, El ambiente. Análisis psicológico. Madrid: Ediciones pirámide.

Durkheim, Émile (1982). El suicidio. Madrid: Akal Universitaria.

Faris,R. (1934). "Cultural isolation and the schizophrenic personality", American J ournal of Sociology, 40, pp.155-169.

Faris, R. y Dunhan, W.H. (1939). Mental disorders in urban areas. Chicago: University of Chicago Press.

Goode, W.J. (1960). "A theory of role strain”, American Sociological Review, 25 pp. 483-496

Gottlieb,B.H. (1981). "Social networks and social support", Studies in Community Mental Health, 4 pp. 304-310.

House, J.S. (1981). "Work, stress and social support", Reading, MA: Addison Wesley

Lin N, ensel WM, Simeone RS, Kuo W. (1979). “Social support stressful life events and illness: a model and empirical test". J. Health Behaviour, 20 pp. 108-119.

Lin N. Dean, A., Ensel WM (1981). "Social support scales: a methodological note". Schizophrenia Bulletin, 1 pp. 73-89.

Marks, S. R. (1977). "Multiples roles and role strain: some notes on human energy, time and commitment", American Sociological Review, Vol. 42 pp. 921-936 Menéndez, E.L. (1984). “El modelo médico hegemónico: transacciones y 


\section{perifèria}

Número 3, Diciembre 2005

www. periferia.name

alternativas hacia una fundamentación teórica del modelo de autoatención en salud", Arxiu d'etnografia de Catalunya, № 3 pp. 85-119.

Requena Santos, F. (2003) "Análisis de redes sociales", Centro de Investigaciones Sociológicas. Siglo XXI.

Sieber, S.D. (1974). "Toward a theory of role accumulation”, American Sociological Review, vol. 39 pp. 567-578.

Shumaker S, Brownell, A. (1984). "Toward a theory of social support: closing conceptual gaps", J. Soc. Issues, 4 pp. 11-36

Thoits, P.A. (1983). "Multiple identities and psychological well-being: a reformulation and test of the social isolation hipotesis", American Sociological review, Vol. 48 pp. 174-187.

Umberson, D (1987). "Family Status and health Behaviors: Social Control as a Dimension of Social Integration", Journal of Health and Social Behavior. Vol. 28 pp.306-319.

Urraca Martínez, S. (1990). “El apoyo social en la salud y en la enfermedad”, J ANO 9-15 Vol XXXVIII № 898 pp. 53

Villalba Quesada, C. (1993). “Redes sociales: Un concepto con importantes implicaciones en la intervención comunitaria", Intervención Psicosocial Vol. II, № 4 pp. 69-85.

Zabalegui Yarnoz, A.; Vidal Milla, A.; Soler Gómez, D.; Latre Méndez, E. (2002) “Análisis empírico del concepto de afrontamiento" Enfermería clínica 2002;12(1) pp. 35- 44 